\title{
Drop-on-Demand System for Manufacturing of Melt-based Solid Oral Dosage: Effect of Critical Process Parameters on Product Quality
}

\author{
Elçin Içten, ${ }^{1,2}$ Arun Giridhar, ${ }^{1}$ Zoltan K. Nagy, ${ }^{1}$ and Gintaras V. Reklaitis ${ }^{1}$
}

Received 28 April 2015; accepted 3 June 2015; published online 17 June 2015

ABSTRACT. The features of a drop-on-demand-based system developed for the manufacture of meltbased pharmaceuticals have been previously reported. In this paper, a supervisory control system, which is designed to ensure reproducible production of high quality of melt-based solid oral dosages, is presented. This control system enables the production of individual dosage forms with the desired critical quality attributes: amount of active ingredient and drug morphology by monitoring and controlling critical process parameters, such as drop size and product and process temperatures. The effects of these process parameters on the final product quality are investigated, and the properties of the produced dosage forms characterized using various techniques, such as Raman spectroscopy, optical microscopy, and dissolution testing. A crystallization temperature control strategy, including controlled temperature cycles, is presented to tailor the crystallization behavior of drug deposits and to achieve consistent drug morphology. This control strategy can be used to achieve the desired bioavailability of the drug by mitigating variations in the dissolution profiles. The supervisor control strategy enables the application of the drop-on-demand system to the production of individualized dosage required for personalized drug regimens.

KEYWORDS: critical process parameters; critical quality attributes; drop-on-demand; drug printing; supervisory control.

\section{INTRODUCTION}

While innovation is the key to success within the pharmaceutical industry, it has been largely sought through new drug discovery and development, while the development of more efficient manufacturing methods has received inadequate attention. Traditionally, the pharmaceutical industry has manufactured its products in large-scale batch processes with nonexistent or limited on-line process monitoring and control. Recently, as a result of encouragement from the regulatory authorities, the advent of globalization, and the increasing awareness of environmental impact, the pharmaceutical industry has been reconsidering the way drug products are developed and manufactured $(1,2)$. The US Food and Drug Administration (FDA) has promoted the Quality by Design (QbD) approach to increase process understanding and improve quality and efficiency while minimizing risk (3). The Process Analytical Technology (PAT) guidance introduced by the FDA encouraged the monitoring of critical quality and performance attributes during processing, with the goal of ensuring final product quality $(4,5)$. As part of this renewed emphasis on improvement of manufacturing, the pharmaceutical industry has begun to develop more efficient

\footnotetext{
${ }^{1}$ School of Chemical Engineering, Purdue University, Forney Hall of Chemical Engineering, 480 Stadium Mall Drive, West Lafayette, Indiana 47907, USA.

${ }^{2}$ To whom correspondence should be addressed. (e-mail: eicten@purdue.edu)
}

production systems with more intensive use of on-line measurement and sensing, real-time quality control and process control tools which offer the potential for reduced production costs, faster product release, reduced variability, increased flexibility and efficiency, and improved product quality (6-8).

Under the US National Science Foundation-supported Engineering Research Center for Structured Organic Particulate Systems, we have developed a dropwise additive manufacturing process for solid oral dosage production. The process utilizes the drop-on-demand (DoD) inkjet printing technology for predictable and highly controllable deposition of active pharmaceutical ingredients (API) onto an edible substrate, such as a polymeric film or placebo tablet $(9,10)$. This process uses fluid operations suitable for low-volume production of personalized dosage forms. The main advantages of the DoD technology are the ability to produce small droplets reproducibly and to print drug formulations with high placement accuracy onto selected substrates (11). The advantages of liquid processing and reproducible production of small droplets create the special opportunity for the production of high-potency, low-dose drugs, which are difficult to produce with consistent quality via conventional powder processing methods. Moreover, the dosage amount can be adjusted according to the patient's needs, by simply changing the drop size or number of drops deposited per dosage. Although different material systems with a wide range of properties can be deposited using DoD technology (12), until recently a limited range of materials have been used in the pharmaceutical inkjet printing applications, namely, solvent- or nano- 
suspension-based formulations $(13,14)$. Using the developed dropwise additive manufacturing process for pharmaceuticals (DAMPP), different drug formulations including solventbased systems, i.e., solvent-polymer-API solutions, as well as melt-based systems, i.e., polymer-API melts, can be printed. Recently, Hirshfield et al. (2014) (9) and Icten et al. (2015) (10) reported proof of concept of dropwise additive manufacturing process for solvent-based applications and for melt-based applications, respectively. Melt-based printing applications eliminate the solvent evaporation step after drop deposition and thus allow on-demand production of individual dosage forms with good control of drug solid state and morphology.

For pharmaceutical processes, the performance of a drug product is defined in terms of its critical quality attributes (CQA), which are its essential physical, chemical, and biological characteristics (15). For the dropwise additive manufacturing system, the desired critical quality attributes (CQA) of the dosage forms that should be kept within the appropriate limits are the dosage amount and drug morphology. The critical process parameters (CPP) that have a direct impact on the CQA's are the drop size, product, and process temperatures, as shown in Table I. This paper presents a supervisory control system implemented on the drop-on-demand manufacturing process which manipulates these CPP's with the goal of ensuring the CQA's are maintained within specified limits. This control system includes on-line monitoring, automation, and closed-loop control, which are essential for producing individual dosage forms with the desired critical quality attributes. In the remainder of the paper, first, the supervisory control framework is presented which serves to control critical process parameters. Then, the effects of process parameters on the final product quality are investigated and the resulting melt-based pharmaceutical dosage forms are characterized using various techniques, including Raman spectroscopy, optical microscopy with a hot stage, and dissolution testing.

\section{SUPERVISORY CONTROL FRAMEWORK}

The prototype dropwise additive manufacturing system is shown in Fig. 1. The system consists of a material reservoir, precision positive displacement pump, xy-staging, a hot airbased heating system, online imaging and sensing, and temperature, pump, and stage controllers. The material reservoir, pump, nozzle, camera, substrate, and staging are labeled in Fig. 1 with numbers 1 through 6 , respectively. The melt-based formulation consists of a low-melting point polymer (or surfactant) carrier and an API, which are co-melted in the material reservoir. This is achieved by heating the reservoir until the polymer melts and the drug dissolves in the molten polymer. The printability and reproducibility of melt formulations are highly dependent on the process temperature, which is maintained above the melting temperature and within the desired operating limits in order to produce melts without causing degradation of the active ingredient. Therefore, temperature control is implemented not only on the reservoir, but also on the pump, tubing, and nozzle using a heating tape, built-in pump heater, and air heating system, respectively. The air heating system consists of concentric tube heat exchanger, in which air is used as the heating medium, and a custom air heater, which is connected to a proportional-integralderivative (PID) temperature controller. The formulation material flows on the tube side, that is, inside the inner tube in the direction shown with the yellow arrow in Fig. 1. The hot air flows on the shell side, that is, around the tubing and the nozzle in the countercurrent direction shown with the blue arrow in Fig. 1. This allows the temperature of the printed formulation to be maintained at the desired setpoint.

The melt-based formulation is precisely deposited onto edible substrates by means of an automation logic, which synchronizes the actions of the pump, camera, and xy-staging components. After a drop is ejected from the nozzle, its image is captured via the camera, which are shown with numbers 3 and 4 in Fig. 1. The dosage amount of each drop is monitored online using the corresponding drop volume calculated using real-time image analysis with arbitrary rotational symmetric shape model (16). Different drop sizes can be produced by changing the pump and nozzle parameters, which enables adjusting the dosage amount for patients with different therapeutic needs. The xy-staging and synchronization logic not only allows precise drop positioning on the substrate while printing but also enables layering of different drugs or of coating materials, thus offering the flexibility of producing combination dosages. In Fig. 2, a photograph of drops deposited on the substrate is shown along with the nozzle and online imaging system, where the substrate is placed above the substrate temperature control system on the xy-staging. Automation of the on-line monitoring and control systems is implemented using the LabVIEW environment. The interested reader is encouraged to refer to Hirshfield et al. (2015) (17) for a more complete exposition of the details of the automated execution of the process and online monitoring system.

The supervisory control framework applied to the dropwise additive manufacturing process is shown in Fig. 3. We developed a network of PID loops controlling the process and product temperatures while executing camera, pump, and staging simultaneously. This framework enables control of the CPP's to achieve the desired CQA's. The dosage amount is determined from the drop size and the known formulation composition. The product solid state depends on the formulation composition, on the selection of the substrate, and on the CPP's, i.e., product temperature and drop size. The selection of the polymer used in the formulation can change the morphology by promoting or inhibiting crystallization of the drug (18). The surface properties of the substrate, such as

Table I. Critical Quality Attributes and Critical Process Parameters

Critical quality attributes (CQA)

Dosage amount

Drug morphology
Critical process parameters (CPP) Drop size

Process temperature

Product temperature 


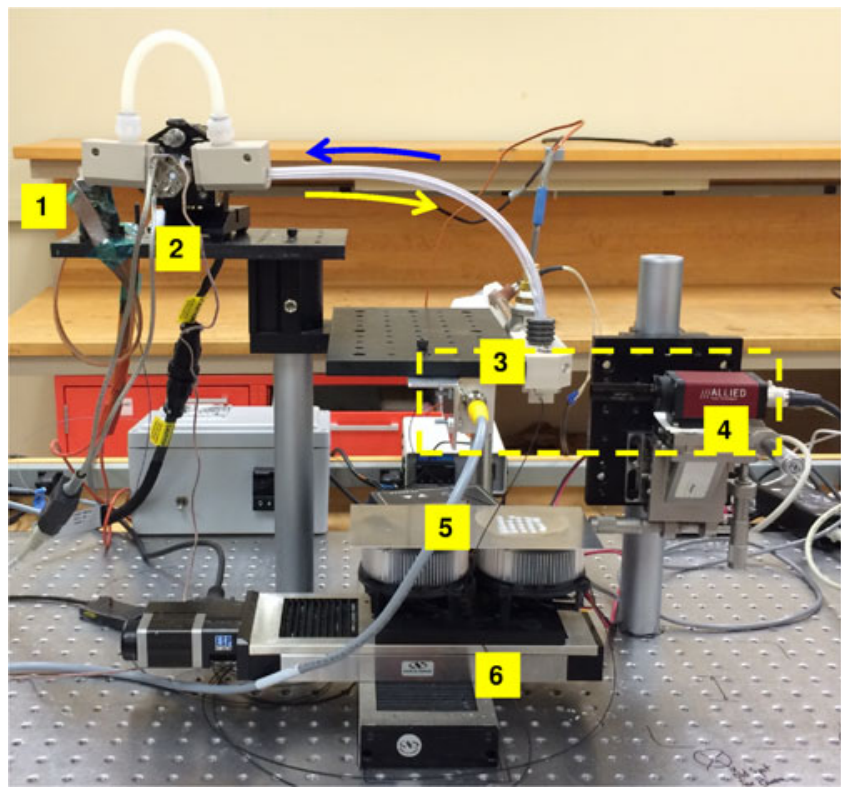

Fig. 1. Dropwise additive manufacturing system ( 1 material reservoir, 2 precision P/D pump, 3 nozzle, 4 camera, 5 substrate, 6 xy-stage, dotted box online imaging system)

roughness and porosity, onto which the drops are deposited also can have an effect on product morphology $(14,19,20)$.

The product temperature corresponds to the crystallization temperature of the deposited drops. The crystallization temperature profile has a strong effect on product solid state and morphology, which influence the dissolution properties and hence the bioavailability of the drug. In bulk crystallization, a controlled temperature profile followed through the crystallization process can affect the final product properties (21-23). Similarly, by manipulating the substrate temperature profile using varying temperature gradients, the drop solidification process can be controlled (24). Since the drop size also affects the drop solidification process by changing the heat transfer dynamics, precise control of the drop solidification process occurring on the substrate is critical. In this process, the crystallization temperature of the deposited drops is controlled via the Peltier devices placed underneath the substrate on the xy-staging. The substrate temperature control via Peltiers is implemented through a PID loop. Programmed temperature gradients, including step changes, ramping heating or cooling, cycling, or any combination of these temperature profiles, can be applied to the drug deposits using the LabVIEW (National Instruments)-based automation of the substrate temperature control system. As in the case of bulk crystallization processes, cycling of temperature also can be an effective mechanism for control of crystal size, thus controlling feature granularity $(25,26)$.

\section{MATERIALS AND METHODS}

\section{Materials and Formulation}

Naproxen was chosen as the model API to form a melt formulation with polyethylene glycol with a molecular weight of 3350. Naproxen (NAP) was purchased from Attix Pharmaceuticals (Montreal, QC, Canada), and PEG 3350 was provided by The Dow Chemical Co. (Midland, MI). Naproxen and PEG 3350 were mixed in a weight ratio of 15:85. The mixture was co-melted at $65^{\circ} \mathrm{C}$ until completely melted. The melt formulation was printed onto polymeric films prepared with hydroxypropyl methylcellulose (HPMC) (E50) and PEG 400. HPMC (E50) was purchased from the Sigma-Aldrich Corporation (St. Louis, MO), and PEG 400 was provided by The Dow Chemical Co. (Midland, MI).

\section{Film Preparation}

The amounts of $0.6 \mathrm{~g}$ HPMC powder and $0.4 \mathrm{~g}$ PEG 400 were dissolved in $20 \mathrm{ml}$ water at $90^{\circ} \mathrm{C}$ to make a $5 \%(w / v)$

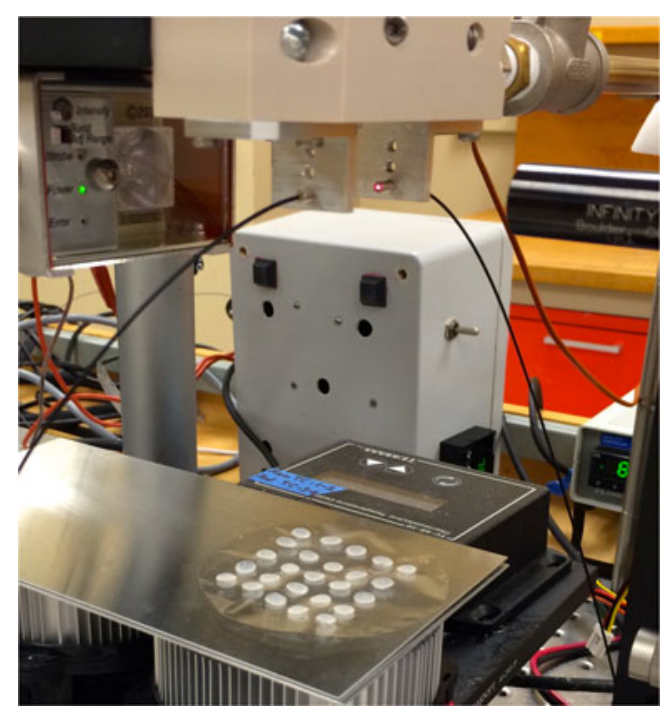

Fig. 2. Melt-based drops (NAP-PEG 3350) deposited on the polymeric film substrate 


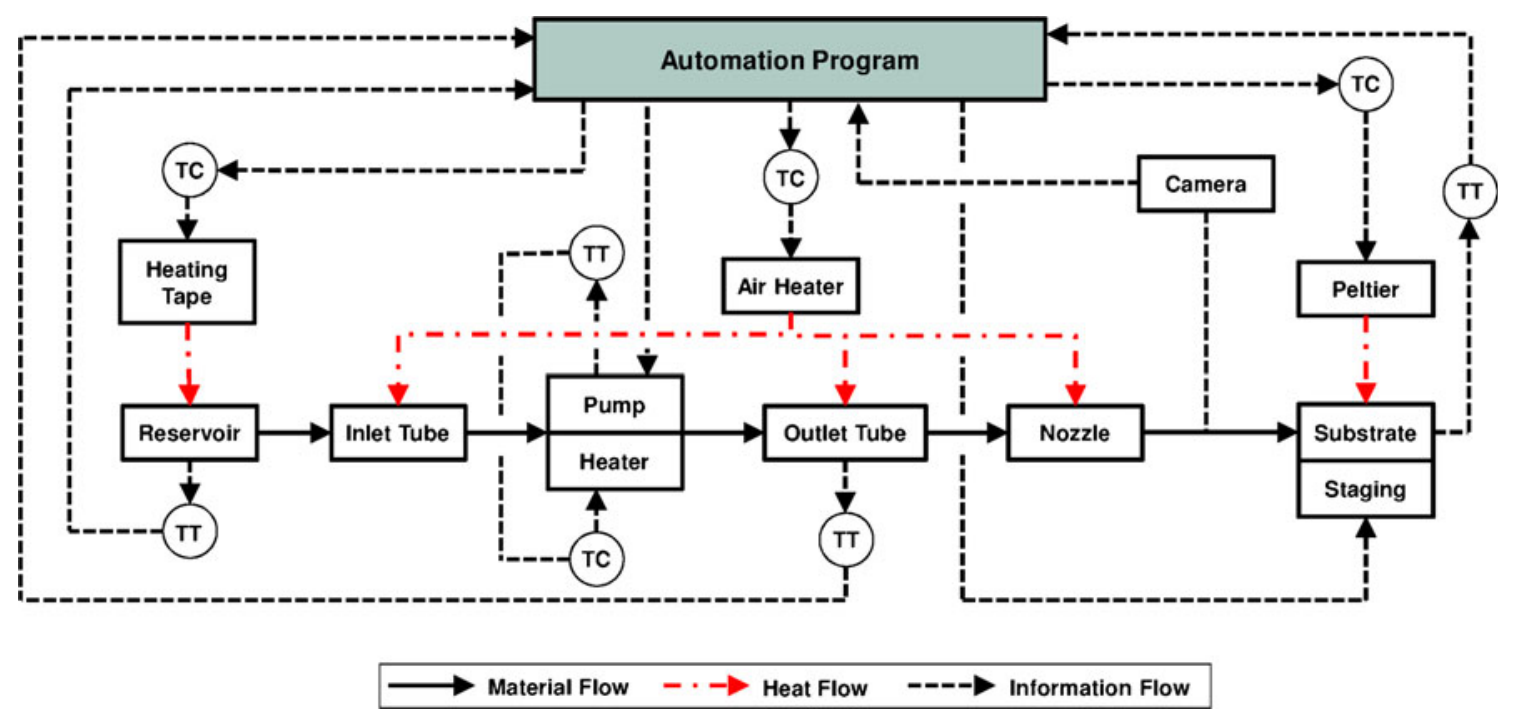

Fig. 3. Supervisory control framework for the dropwise additive manufacturing process

polymer solution. The 5\% (w/v) HPMC-PEG 400 solution was stirred overnight at room temperature to ensure that the polymeric chain was homogeneously dispersed in the solution and cast onto a Petri glass. After drying was completed, the film was peeled off.

\section{Methodology}

After printing of the drops onto the film, the resulting dosage forms were analyzed to determine whether and to what extent the different process parameters affect the dissolution behavior and solid-state characteristics of the API. Since the dosage forms were created and analyzed within a matter of minutes, varying ambient conditions, such as relative humidity, were not impactful on the results.

\section{Reproducibility of Drop Sizes}

Two different drop sizes are used to produce the dosage forms with target dosage of $15 \mathrm{mg}$ of API. The different drop sizes are obtained by changing the pump settings such as displacement, volume strokes, and rate as well as nozzle size. For simplicity, the drops with different sizes will be referred as "small" and "large" drops. To analyze reproducibility, HPMCPEG 400 film measuring $2 \mathrm{~cm}$ by $2 \mathrm{~cm}$ was weighed on an Omega AL-201s balance and a specific number of drops were deposited on the film to reach the target dosage amount. Before producing the dosage forms, the number of drops needed to reach the target amount varied for each printing setting was determined experimentally. The films were then subjected to different cooling temperature profiles to cause solidification and crystallization. Following the solidification of the drops, the films were weighed again to determine the total mass of the deposits on the film. Içten et al. (2015) (10) confirmed using high-performance liquid chromatography (HPLC) experiments that the compositions of the deposits were identical to the composition of the drug formulation in the melt reservoir. Therefore, the amount of drug in each drop can be precisely determined by multiplying the mass of solids by the percentage of drug in the solution $(15 \%)$. These results were then used to analyze how consistently and accurately the drop-on-demand system creates dosage forms.

\section{Raman Microscopy and Mapping}

A Raman RXN1 Microprobe (Kaiser Optical Systems, MI) was used to analyze the crystal structure of the melt formulations and to build a map of the drop deposits. First, the spectra of pure naproxen and PEG 3350 solid dispersions were obtained. Naproxen and PEG 3350 powders were heated above their melting temperatures, to 160 and $60^{\circ} \mathrm{C}$, respectively. The melts of pure naproxen and pure PEG 3350 were solidified at room temperature and then analyzed to obtain the spectra of pure compounds. The spectra of the melt formulation consisting of 15\% naproxen and 85\% PEG 3350 were obtained by analyzing the drops of the melt formulation deposited using the dropwise additive manufacturing process. Raman mapping of the dosage forms was created to study the distribution of the drug over the deposited drops. Therefore, a large area of the drop, $660 \mu \mathrm{m} \times 1000 \mu \mathrm{m}$ was mapped with $100 \mu \mathrm{m}$ step sizes, and the map was taken in several different areas of the drop The ratio of the characteristic peaks of the drug to polymer were used in building the color intensity map.

\section{Hot-Stage Microscopy}

A Zeiss Axio Imager A2m polarized light microscope (Carl Zeiss Microscopy, LLC, NY) equipped with a Linkam THMS 600 hot-stage (Linkam Scientific Instruments Ltd., Surrey, UK) was used for this study. The naproxen and PEG 3350 were physically mixed in (15:85) weight ratio and heated to $65^{\circ} \mathrm{C}$ until completely melted. After a homogeneous melt was formed, it was cooled down to $30^{\circ} \mathrm{C}$ with the different cooling rates shown in section "Effect of Crystallization Temperature Control on Product Solid State." The induction points were determined by recording the temperature of the sample when the first nucleus was observed. The measurements were taken in two replicates in order to determine the mean induction temperatures and times and their standard deviations. 


\section{Dissolution Testing}

The dissolution tests were conducted using a USP-I apparatus (Varian VK 7010, Agilent Technologies, Santa Clara, CA) operated at $100 \mathrm{rpm}$. The dissolution media consisted of the USP buffer solution of $\mathrm{pH} 7.4$, which was maintained at $37^{\circ} \mathrm{C}$. Experiments were run for $90 \mathrm{~min}$, and aliquots of the dissolution medium were collected at intervals of $3 \mathrm{~min}$ through $35 \mu \mathrm{m}$ full flow filters (Agilent filters) by a peristaltic pump. Sample absorbance was measured by a UV spectrophotometer (Agilent Cary 50) at $243 \mathrm{~nm}$. Absorbance values from the spectrophotometer were used to calculate the percent release of the API from the films. Each experiment was performed in three replicates.

\section{RESULTS AND DISCUSSION}

In order to study the effects of critical process parameters on the product solid state and morphology, dosage forms of melts were produced using two different drop sizes by applying different substrate temperature profiles. The melt formulation consisted of $15 \%$ naproxen and $85 \%$ polyethylene glycol 3350 (PEG 3350) by weight. This formulation was selected based on the studies done by Zhu et al. (2013) which showed that naproxen and PEG 3350 form a eutectic mixture at a composition of $15 \%$ naproxen (27). The formulation was co-melted at $65^{\circ} \mathrm{C}$, at which temperature the polymer melts and the drug dissolved in the molten polymer. The temperature of the system was controlled at $70^{\circ} \mathrm{C}$ throughout the process. The molten formulation was subjected to the operating temperature of $70^{\circ} \mathrm{C}$ during the maximum residence time of $5.4 \mathrm{~min}$, which was achieved with the slowest production rate. In our previous study, the chemical stability of naproxen during production was investigated via HPLC experiments, which showed that the drug found in the dosage forms was stable for at least 15 min under the same operating conditions (10).

Using the DoD manufacturing system, dosage forms containing $15 \mathrm{mg}$ of naproxen are produced. The reproducibility of the produced dosage forms is shown in Table II. Good reproducibility with as low as $2 \%$ relative standard deviation can be achieved for melt-based pharmaceuticals produced with this process. The number of drops to be printed on the HPMC-PEG films was adjusted depending on the drop size to reach the proper dosage amount. Dosage forms were produced by printing either "large" drops of size $23.4 \mathrm{mg}$ with a standard deviation of $1.4 \mathrm{mg}$ or "small" drops of size $19.4 \mathrm{mg}$ with a standard deviation of $0.3 \mathrm{mg}$ on HPMC-PEG films as the substrate.

The crystallinity of the melt formulations consisting of 15\% naproxen and $85 \%$ PEG 3350 was studied using Raman microscopy. Raman spectra of pure naproxen melt, pure PEG 3350 melt, and melt-based drug deposits of NAP-PEG 3350 $(15: 85)$ are presented in Fig. $4 \mathrm{a}, \mathrm{b}, \mathrm{c}$, respectively. The characteristic peaks of pure naproxen and pure PEG 3350 at 760 and $1280 \mathrm{~cm}^{-1}$ are used for the analysis. Raman spectra of the dosage forms confirm that naproxen present in the dosage forms is crystalline, which is in accordance with x-ray diffraction analysis of the same formulation reported by Icten et al. (2015) (10).

The drug distribution throughout the deposited drop is analyzed using Raman mapping employed for the dosage forms. Different areas throughout the drop deposits were analyzed (data not shown). A color intensity Raman map was built based on the ratio of the characteristic peaks of naproxen and PEG 3350 at 760 and $1280 \mathrm{~cm}^{-1}$, respectively. In Figure 5, a representative area $(660 \mu \mathrm{m} \times 1000 \mu \mathrm{m})$ of the drop deposits is mapped. The small relative intensity differences confirm that naproxen has an even distribution throughout the drop. This finding is in accordance with HPLC analysis conducted on the melt-based formulations by Içten et al. (2015), which suggested that the amount of drug recovered from each drop was the same as the amount present in the drug formulation (10). Here, we show that in a sample area of the drop, the drug is distributed well within the polymer matrix. Raman measurements performed over different areas of the droplet indicated similarly homogenous drug distribution.

\section{Effect of Crystallization Temperature Control on Product Solid State}

Since the crystallization temperature of the drug deposits has an effect on the product solid state and morphology, precise control of the drop crystallization and solidification processes is crucial to reach the desired product quality. In our DOD system, different programmed cooling temperature profiles were applied to the substrates containing the drug deposits. Specifically, in this study, we designed four different temperature profiles and applied them via the Peltier devices placed underneath the substrate. The molten deposits were cooled from $60^{\circ} \mathrm{C}$ down to $30^{\circ} \mathrm{C}$ using different temperature profiles, which are shown in Fig. 6. A constant temperature profile is achieved by printing the drops onto films maintained at $30^{\circ} \mathrm{C}$ and by controlling its temperature at $30^{\circ} \mathrm{C}$ until the drops are solidified. The other dosage forms were printed onto films maintained at $60^{\circ} \mathrm{C}$ and cooled down to $30^{\circ} \mathrm{C}$ using a fast cooling rate of $10^{\circ} \mathrm{C} / \mathrm{min}$ or a slow cooling rate of $1^{\circ} \mathrm{C} /$ min or by applying heating/cooling cycles where the dosage forms were cooled with repeated cycles of cooling with a rate of $10^{\circ} \mathrm{C} / \mathrm{min}$ for $1 \mathrm{~min}$ followed by heating with a rate of $1^{\circ} \mathrm{C} /$ min for 1 min until the deposit reached $30^{\circ} \mathrm{C}$.

In order to study the crystallization of the drug within the dosage forms under different temperature profiles, optical microscopy experiments are performed with a hot stage following the cooling temperature profiles shown on Fig. 6. Although there were no differences observed in the crystallinity

Table II. Reproducibility Analysis of Dosage Forms

\begin{tabular}{lll}
\hline Drop size & Average dosage amount $(\mathrm{mg})$ & RSD (\%) \\
\hline Small & 14.56 & 1.71 \\
Large & 14.27 & 5.74 \\
\hline
\end{tabular}



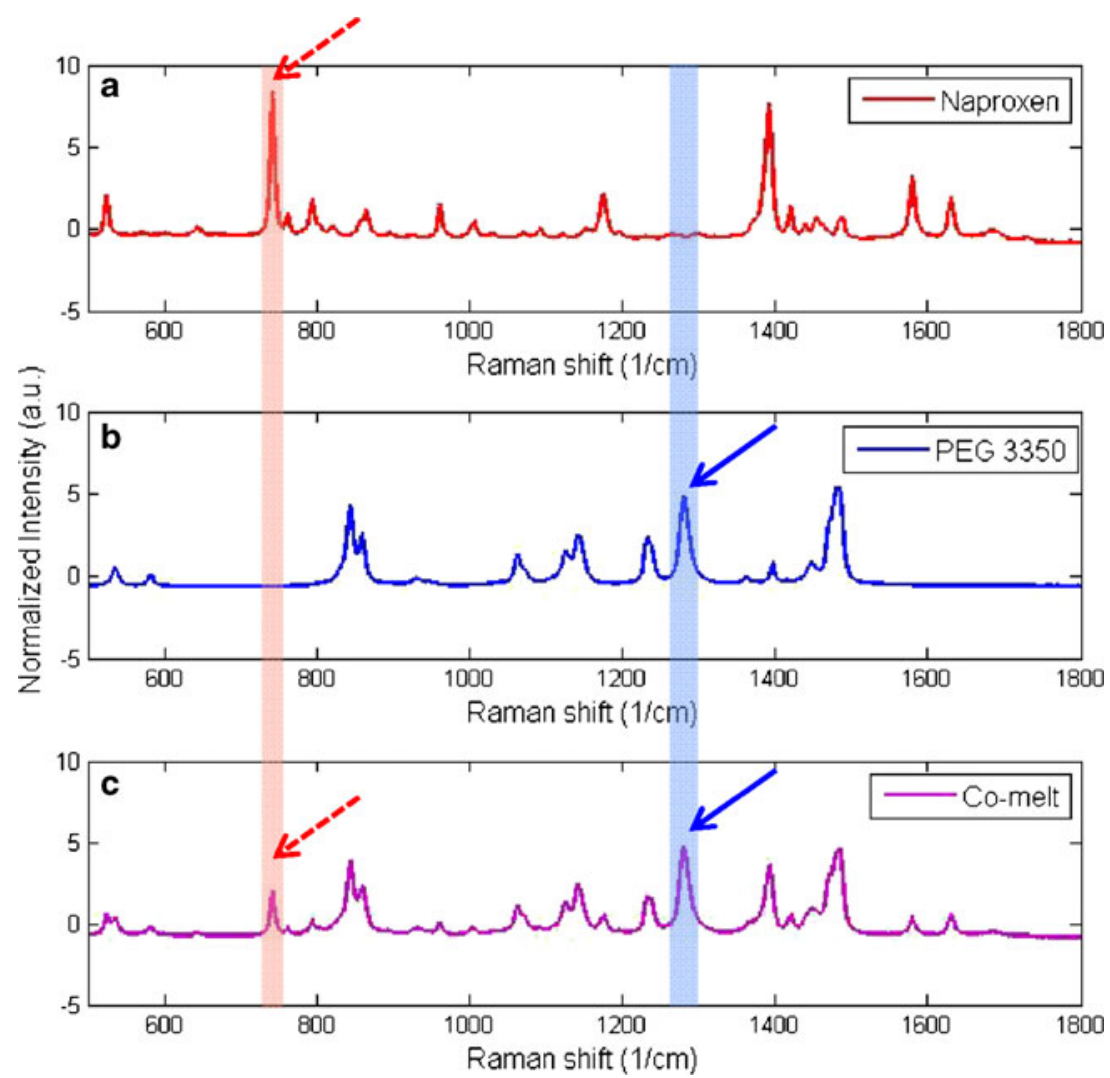

Fig. 4. Raman spectra of a pure NAP, b pure PEG 3350, c co-melt of NAP-PEG 3350 (15:85). Characteristic peaks at 760 and $1280 \mathrm{~cm}-1$ are shown with red and blue arrows, respectively

of the dosage forms, the application of different temperature profiles did in fact change the crystallization behavior and morphology. The induction times for crystal formation showed differences based on the temperature profile applied. Using the images recorded via hot-stage microscopy, the induction time and temperatures corresponding to the first nucleus formation were determined. In Fig. 6, the induction times are shown with the points marked on the fast cooling, slow cooling, and cycling temperature profile curves. The measurements were taken in replicates to determine the mean induction temperatures and times, and the corresponding standard deviations are listed in Table III. Under the fast cooling profile, the induction occurs at $36.6^{\circ} \mathrm{C}$ in $4.3 \mathrm{~min}$. Under cycling and slow cooling temperature profiles, the average induction temperatures are 42.0 and $45.6^{\circ} \mathrm{C}$ occurring in 6.4 and $16.4 \mathrm{~min}$, respectively. These results indicated that instead of

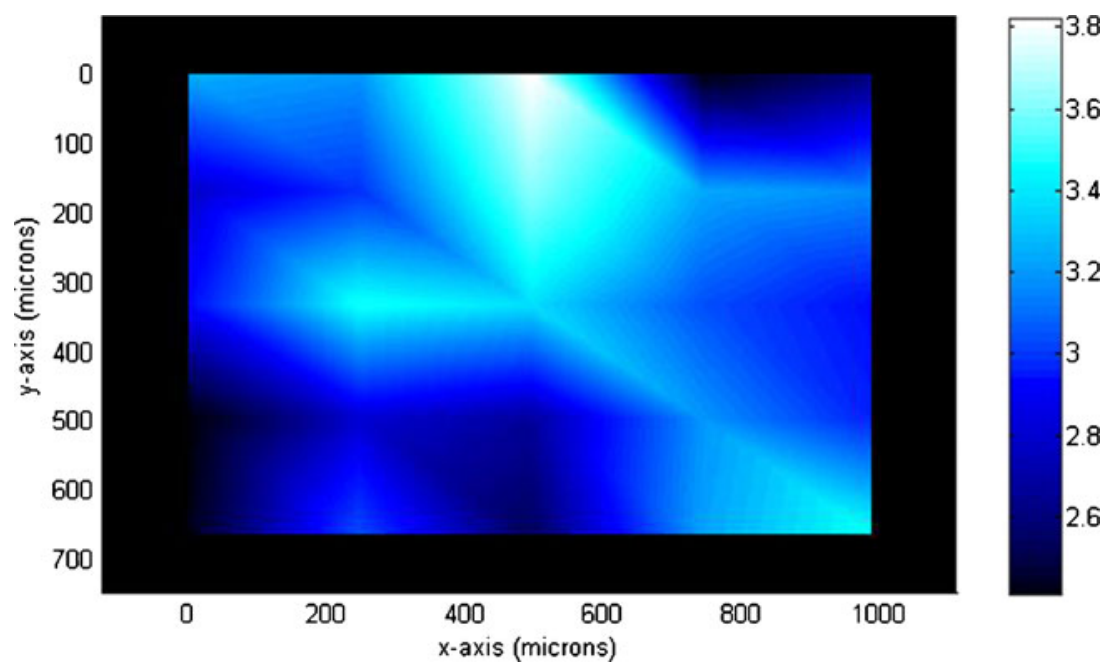

Fig. 5. Raman map of melt-based deposits of NAP-PEG 3350 (15:85). Map area $660 \mu \mathrm{m} \times 1000 \mu \mathrm{m}$ 


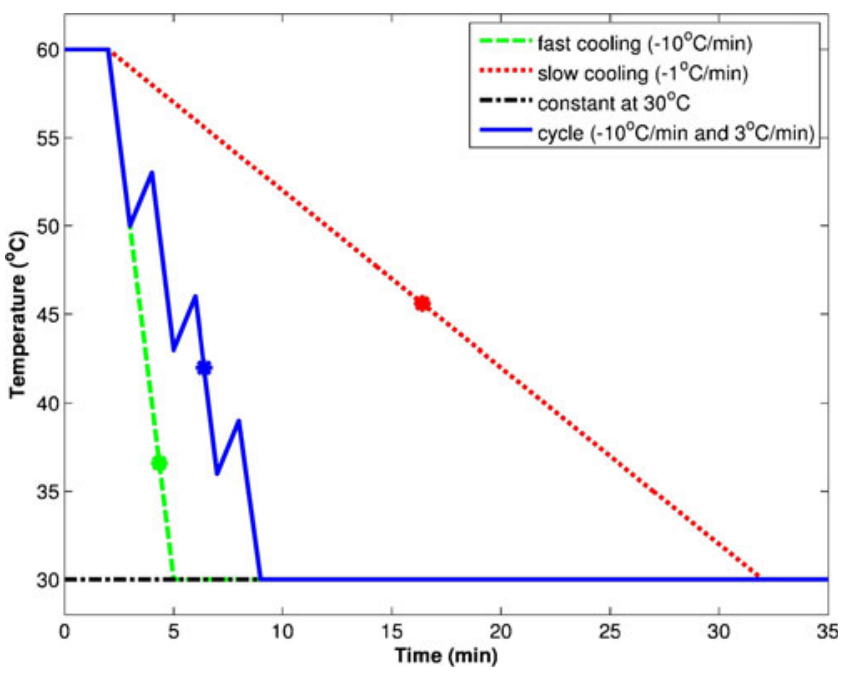

Fig. 6. Temperature profiles applied on the substrate

applying slow cooling for 16.4 min, a designed temperature cycle can be applied to shorten the induction time by keeping the induction temperature, hence crystallization behavior, similar.

In addition to the differences observed in the crystallization behavior, morphological differences are also observed between the dosages solidified under different temperature profiles using optical microscopy with a hot stage. When the melts are cooled down with the fast cooling rate of $10^{\circ} \mathrm{C} / \mathrm{min}$, surface defects are observed, which are shown in Fig. 7a. This is mainly due to the fact that fast cooling resulted in more nucleation sites during crystallization, thus producing higher surface area of crystals. When the melts are cooled down with the slow cooling rate of $1{ }^{\circ} \mathrm{C} / \mathrm{min}$, surface defects were reduced; however, they were still observed as shown in Fig. 7b. When the melts were subjected to the cycling temperature profile, the surface defects were eliminated, as shown in Fig. 7b. With designed cycles and through repeated partial re-melting and solidifying the dosage forms, alternative morphology changes can be obtained.

\section{Effect of Critical Process Parameters on the Dissolution of Dosage Forms}

The crystallization temperature profile applied to the drug deposits affects both the crystallization behavior and the morphology of the drug, which are known to influence both the dissolution behavior and bioavailability of the drug. Therefore, we performed dissolution testing to study the effect of the substrate temperature profile and also the effect of the drop size on the dissolution properties of the drug. Dosage forms of melts containing 15\% naproxen and 85\% PEG 3350 were produced using small and large drop sizes by applying different substrate temperature profiles.
First, we studied the effect of substrate temperature profile and compared the dissolution behaviors of the dosage forms produced with the same drop size and following different cooling profiles. The dissolution profiles of the dosage forms, which are produced with small drop size and solidified using fast or slow cooling rates, are compared in Fig. 8a. When the fast cooling rate of $10^{\circ} \mathrm{C} / \mathrm{min}$ is applied to the dosage forms containing small drops, faster dissolution is observed than is seen with samples produced with the slower cooling rate of $1^{\circ} \mathrm{C} / \mathrm{min}$. The dissolution profiles of the dosage forms, which are produced with large drop size and solidified using fast or slow cooling rates, are compared in Fig. 8b. Similar to the behavior of the dosage forms containing small drops, faster dissolution is observed when the fast cooling rate of $10^{\circ} \mathrm{C} / \mathrm{min}$ is applied to the dosage forms containing large drops. During fast cooling, more nucleation sites are created that result in higher surface area, which was also observed with hot-stage microscopy experiments, and therefore in faster dissolution of the dosage forms.

We further investigated the effect of the drop size and compared the dissolution behavior of the dosage forms containing either large or small drops under the same crystallization temperature profiles. When the temperature of the drug deposits containing either small or large drops are controlled at a constant temperature of $30^{\circ} \mathrm{C}$, significant variation of the dissolution profiles are observed both within the dosage forms of the same drop size and between the dosage forms containing different drop sizes. This variation is evident from the error bars shown in Fig. 9a. When the substrate temperature is held constant, then the cooling profile within the droplets is influenced by their volume, which can result in significant variations in the crystallization conditions. While control of the temperature of the deposits was exercised by manipulating the temperature of the surface of the Peltier, the temperature within a deposit is non-uniform. Indeed, infrared camera

Table III. Induction Points When Different Temperature Profiles are Applied

\begin{tabular}{llll}
\hline & Fast cooling & Cycling & Slow cooling \\
\hline Induction temperature $\left({ }^{\circ} \mathrm{C}\right)$ & $36.6 \pm 1.5$ & $42.0 \pm 0.8$ & $45.6 \pm 1.5$ \\
Induction time $(\min )$ & 4.3 & 6.4 & 16.4 \\
\hline
\end{tabular}



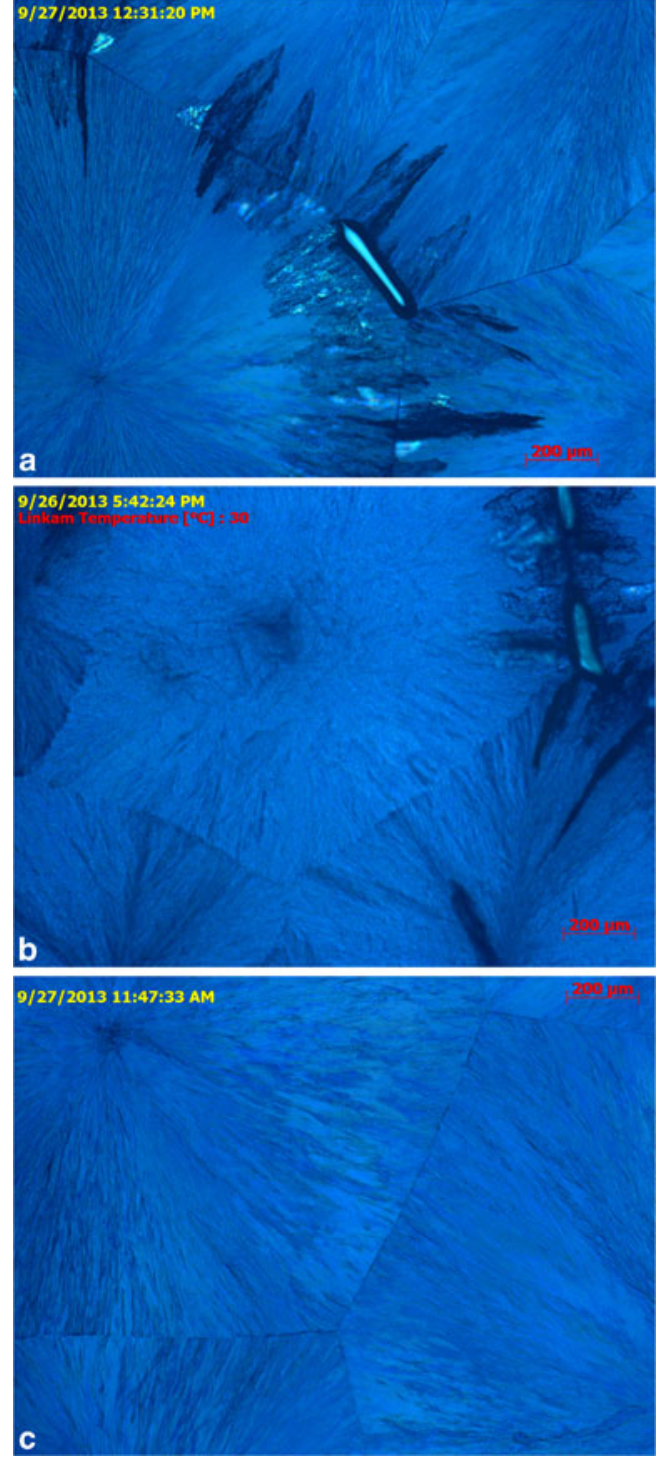

Fig. 7. Optical microscopy images of melt-based deposits (NAP-PEG 3350) after a fast cooling b slow cooling $\mathbf{c}$ cycling

images of melt-deposits solidifying at room temperature, shown in Fig. 10, indicate that there are temperature gradients present within the drops. However, when the drug deposits are solidified by applying a fast cooling rate of $10^{\circ} \mathrm{C} / \mathrm{min}$, the temperature gradient within the dosages containing the same drop size decreases significantly. Thus, this results in smaller variation in the dissolution profile for the dosage forms containing the same size of droplets, which reflected in the reduced error bars shown in Fig. 9b. In the case of solidification of dosage forms by applying a slow cooling rate of $1^{\circ} \mathrm{C} / \mathrm{min}$, the differences in the dissolution profile of the dosages containing both the same drop size and different drop sizes decrease further, as shown in Fig. 9c. The use of the slow cooling profile minimizes the differences due to drop size by enabling better heat transfer and thus minimizes the spatial distribution of temperature differences.

Finally, the effect of cycling of the substrate temperature on the dissolution profile was studied. The effect of the drop sizes used can be seen in terms of the different dissolution
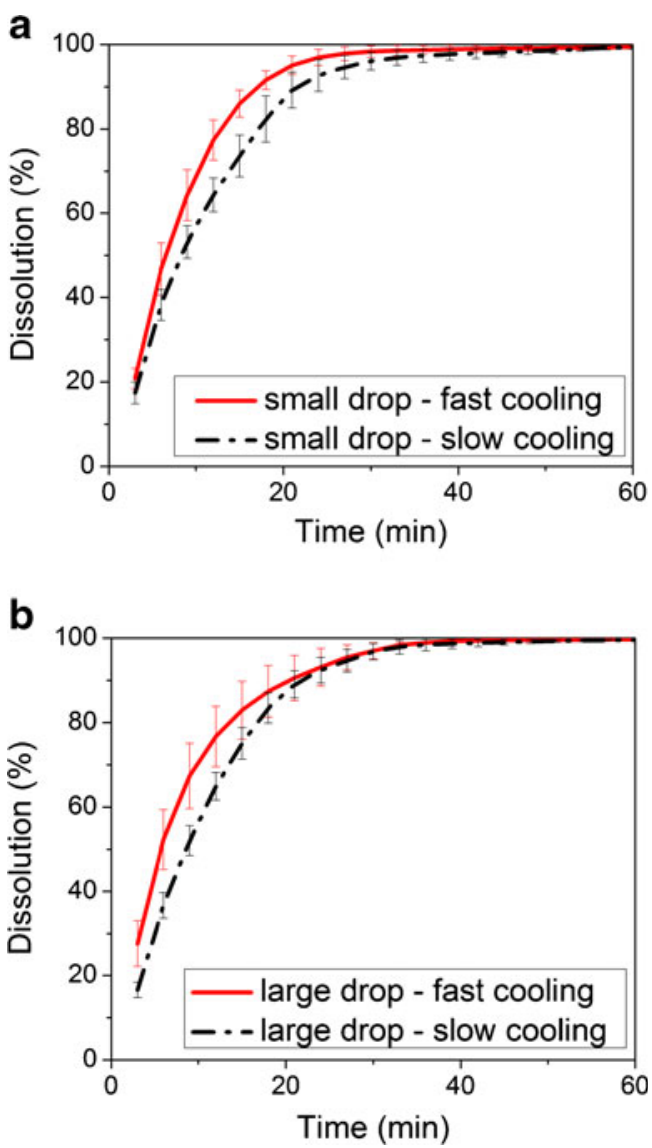

Fig. 8. Dissolution profiles of the dosage forms solidified with different cooling rates. a Dosage forms containing small drops. b Dosage forms containing large drops

rates. There are small variations in the dissolution of the dosages produced with the same drop size as represented by reduced error bars in Fig. 10d. Cycling of temperature is also an effective mechanism for the control of crystal morphology as further discussed in section "Effect of Crystallization Temperature Control on Product Solid State.” Designed cycles can be used to eliminate surface defect and achieve morphological changes by repeated partial re-melting and solidifying the dosage forms. It can also be used to reduce the time for crystallization and achieve a similar crystallization behavior.

Since pharmaceutical products must meet the target bioavailability regardless of their dosage amount, knowledge of the effect of the process conditions on the dissolution of the drug is of utmost importance. Thus, by applying an appropriate cooling profile, the differences due to drop sizes can be minimized and a desired dissolution rate can be achieved for dosages with different drop sizes.

With the model formulation used in this study, the amorphous form of naproxen is not produced in the presence of PEG 3350, since it actually promotes crystallization of naproxen. However, amorphous forms can be produced with alternative choice of polymers, which inhibit crystallization, along with suitable choice of operating conditions. It is well known that the dissolution of low-solubility drugs can be enhanced, by producing product forms in which the active is in amorphous forms. However, since stability of amorphous drug forms can be challenging, design of crystallization 
a

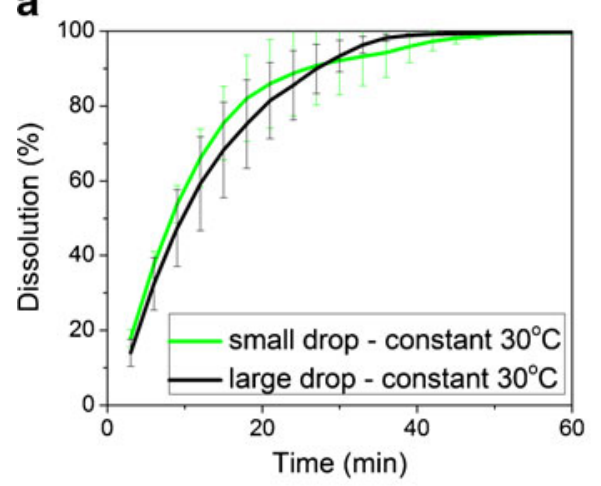

b

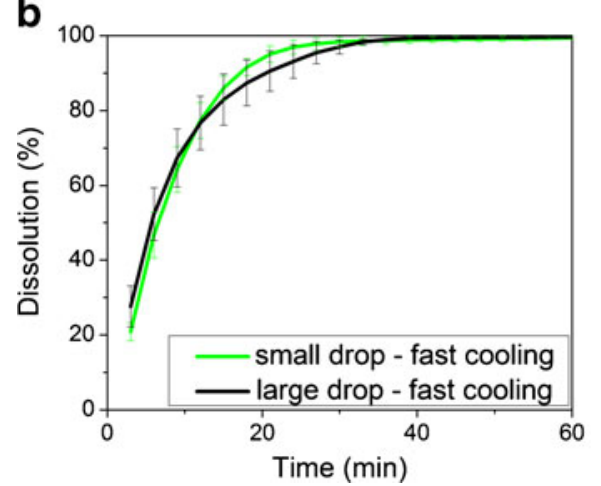

C

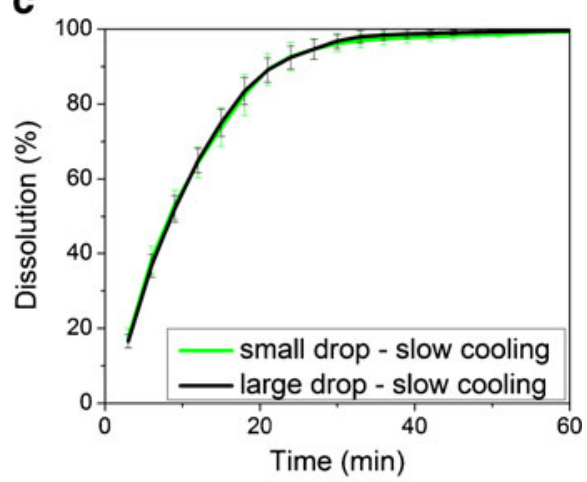

d

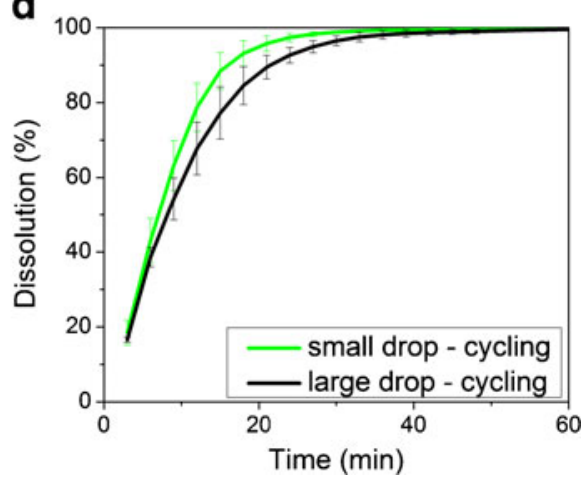

Fig. 9. Dissolution profiles of the dosage forms created with two different drop sizes and solidified at different temperature profiles a constant temperature, $\mathbf{b}$ fast cooling, $\mathbf{c}$ slow cooling, $\mathbf{d}$ cycling

temperature profile and precise control of substrate temperature profile are even more important than in the case of crystalline API formulations. Controlled production with amorphous forms with the drop-on-demand system is the subject of ongoing research.

\section{CONCLUSIONS}

A dropwise additive manufacturing process has been developed for the production of melt-based solid oral dosages. In this paper, a supervisory control system implemented for the dropwise additive manufacturing process is reported which has as its goal ensuring reproducible production and

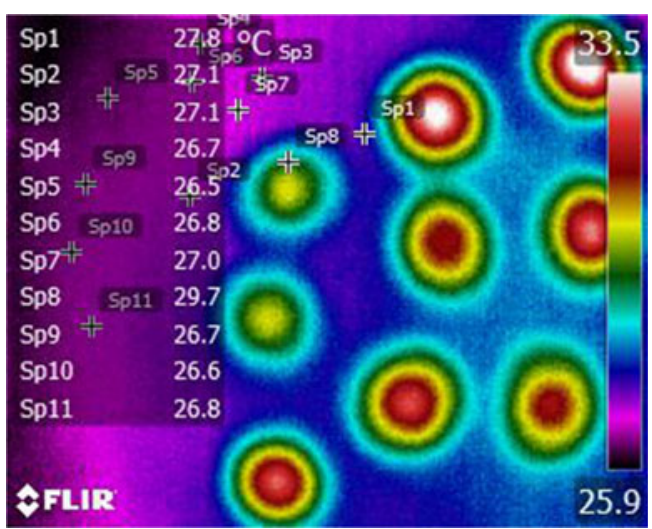

Fig. 10. Infrared camera image of melt-based deposits solidifying at room temperature final product quality. The effect of the critical process parameters, such as drop size and product and process temperatures, on the final product quality, namely dosage amount and drug morphology, is investigated and the produced melt-based pharmaceutical dosage forms are analyzed. Dosage forms of melts containing the model formulation of naproxen and PEG were produced using small and large drop sizes by following selected substrate temperature profiles, including cooling ramping profiles, constant temperature, and temperature cycling. The presented crystallization temperature control strategy is used to tailor the crystallization behavior of drug deposits and to achieve consistent drug morphology. Hotstage microscopy studies prove that the different product morphologies can be achieved by controlling the cooling profile. Our results indicate that by applying controlled temperature cycles on the deposits, the desired drug morphology and crystallization behavior can be achieved. Moreover, meltbased dosages of smaller drops have faster dissolution compared to melt-based dosages of larger drops with the same dosage amount. Thus, the supervisory control strategy can be used to monitor the drop size online and to predict a crystallization temperature profile for the monitored drop size such that the desired bioavailability of the drug is achieved and variations in the dissolution profiles due to variable dosage amount are mitigated. Hence, it enables the application of the drop-on-demand system for the production of individualized dosage regimens for personalized treatments.

Although the use of PEG in the formulation enhances the dissolution of naproxen, the API present in the melt-based formulation is in crystalline form. Solubility of APIs can be enhanced further by using the active ingredient in the 
amorphous form. Future goals include expanding our work to a melt-based formulation, where the API is found in the amorphous form. Amorphous forms can be stabilized through designed temperature profiles, which can be then controlled and applied with the supervisory control system.

\section{ACKNOWLEDGEMENTS}

This work was funded by the National Science Foundation under grant EEC-0540855 through the Engineering Research Center for Structured Organic Particulate Systems. The authors would like to thank Indiana Next Generation Manufacturing Competitiveness Center (IN-MaC) for financial support provided to Elçin Içten. The authors would also like to thank Golshid Kevyan for her help with the dissolution analysis of the dosage forms.

\section{REFERENCES}

1. Cervera-Padrell AE, Skovby T, Kiil S, Gani R, Gernaey KV. Active pharmaceutical ingredient (API) production involving continuous processes-a process system engineering (PSE)-assisted design framework. Eur J Pharm Biopharm. 2012;82(2):437-56.

2. Gupta A, Giridhar A, Venkatasubramanian V, Reklaitis GV. Intelligent alarm management applied to continuous pharmaceutical tablet manufacturing: an integrated approach. Ind Eng Chem Res. 2013;52(35):12357-68.

3. Yu LX, Amidon G, Khan MA, Hoag SW, Polli J, Raju GK, et al. Understanding pharmaceutical quality by design. AAPS J. 2014;16(4):771-83.

4. Food and Drug Administration CDER. Guidance for industry PAT - a framework for innovative pharmaceutical. 2004.

5. Simon LL, Pataki H, Marosi G, Meemken F, Hungerbu K, Baiker A, et al. Assessment of recent process analytical technology (PAT) trends: a multiauthor review. Org Process Res Dev. 2015;19(1):3-62.

6. Stephanopoulos G, Reklaitis GV. Process systems engineering: from Solvay to modern bio- and nanotechnology. Chem Eng Sci. 2011;66(19):4272-306.

7. Gernaey KV, Cervera-Padrell AE, Woodley JM. A perspective on PSE in pharmaceutical process development and innovation. Comput Chem Eng. 2012;42:15-29.

8. Troup GM, Georgakis C. Process systems engineering tools in the pharmaceutical industry. Comput Chem Eng. 2013;51:157-71.

9. Hirshfield L, Giridhar A, Taylor LS, Harris MT, Reklaitis GV. Dropwise additive manufacturing of pharmaceutical products for solvent-based dosage forms. J Pharm Sci. 2014;103(2):496-506.

10. Içten E, Giridhar A, Taylor LS, Nagy ZK, Reklaitis GV. Dropwise additive manufacturing of pharmaceutical products for melt-based dosage forms. J Pharm Sci. 2015;104(5):1641-9.
11. de Gans B-J, Duineveld PC, Schubert US. Inkjet printing of polymers: state of the art and future developments. Adv Mater. 2004;16(3):203-13.

12. Derby B. Inkjet printing of functional and structural materials: fluid property requirements, feature stability, and resolution. Annu Rev Mater Res. 2010;40(1):395-414.

13. Alomari M, Mohamed FH, Basit AW, Gaisford S. Personalised dosing: printing a dose of one's own medicine. Int J Pharm. 2014. doi:10.1016/j.ijpharm.2014.12.006.

14. Kolakovic R, Viitala T, Ihalainen P, Genina N, Peltonen J, Sandler N. Printing technologies in fabrication of drug delivery systems. Expert Opin Drug Deliv. 2013;10(12):1711-23.

15. Food and Drug Administration CDER. Q8 (R1) Pharmaceutical development revision 1. 2008.

16. Hugli H, Gonzalez J. Drop volume measurements by vision. Imaging 2000, SPIE Electron Imaging Conf San Diego. 2000;3966(11):60-6.

17. Hirshfield L, Içten E, Giridhar A, Nagy ZK, Reklaitis GV. Realtime process management strategy for dropwise additive manufacturing of pharmaceutical products. J Pharm Innov. 2015. doi:10.1007/s12247-015-9218-5.

18. Trasi NS, Taylor LS. Effect of polymers on nucleation and crystal growth of amorphous acetaminophen. CrystEngComm. 2012;14(16):5188.

19. Hsu H, Toth SJ, Simpson GJ, Taylor LS, Harris MT. Effect of substrates on naproxen-polyvinylpyrrolidone solid dispersions formed via the drop printing technique. J Pharm Sci. 2013;102(2):638-48.

20. Sandler N, Ihalainen P, Kronberg L, Meierjohann A, Viitala T, Peltonen J. Inkjet printing of drug substances and use of porous substrates-towards individualized dosing. J Pharm Sci. 2011;100(8):3386-95.

21. Acevedo D, Nagy ZK. Systematic classification of unseeded batch crystallization systems for achievable shape and size analysis. J Cryst Growth. 2014:394:97-105.

22. Nagy ZK, Braatz RD. Advances and new directions in crystallization control. Annu Rev Chem Biomol Eng. 2012;3:5575.

23. Fujiwara M, Nagy ZK, Chew JW, Braatz RD. First-principles and direct design approaches for the control of pharmaceutical crystallization. J Process Control. 2005;15(5):493-504.

24. Icten E, Nagy ZK, Reklaitis GV. Supervisory control of a drop on demand mini-manufacturing system for pharmaceuticals. Proc 24th Eur Symp Comput Aided Process Eng. 2014;33:535-40.

25. Abu Bakar MR, Nagy ZK, Rielly CD. Seeded batch cooling crystallization with temperature cycling for the control of size uniformity and polymorphic purity of sulfathiazole crystals. 2009;(3):1343-56.

26. Abu Bakar MR, Nagy ZK, Rielly CD. Investigation of the effect of temperature cycling on surface features of sulfathiazole crystals during seeded batch cooling crystallization. Cryst Growth Des. 2010;10(9):3892-900.

27. Zhu Q, Toth SJ, Simpson GJ, Hsu H-Y, Taylor LS, Harris MT. Crystallization and dissolution behavior of naproxen/ polyethylene glycol solid dispersions. J Phys Chem B. 2013;117(5):1494-500. 